\title{
Taylor-Couette Flow of an Oldroyd-B Fluid in an Annulus Subject to a Time-dependent Rotation
}

\author{
M. Imran', Madeeha Tahir', M. A. Imran', A. U. Awan ${ }^{3}$ \\ ${ }^{1}$ Department of Mathematics, Government College University, Faislabad, Pakistan \\ ${ }^{2}$ School of Science and Technology, Department of Mathematics, University of Management and Technology Lahore, Lahore, Pakistan \\ ${ }^{3}$ Department of Mathematics, University of the Punjab, Lahore, Pakistan
}

\section{Email address:}

drmimranchaudhry@gmail.com (M. Imran),dr.madeeha.2017@gmail.com (M. Tahir), imranasjad11@yahoo.com (M. A. Asjad), auawan2003@yahoo.com (A. U. Awan)

\section{To cite this article:}

M. Imran, Madeeha Tahir, M. A. Imran, A. U. Awan. Taylor-Couette Flow of an Oldroyd-B Fluid in an Annulus Subject to a Time-dependent Rotation. American Journal of Applied Mathematics. Special Issue: Proceedings of the 1st UMT National Conference on Pure and Applied Mathematics (1st UNCPAM 2015). Vol. 3, No. 3-1, 2015, pp. 25-31. doi: 10.11648/j.ajam.s.2015030301.15

\begin{abstract}
In this paper the velocity field and the adequate shear stress corresponding to the rotational flow of an Oldroyd-B fluid, between two infinite coaxial circular cylinders, are determined by applying the finite Hankel transforms. The motion is produced by the inner cylinder that, at time $\mathrm{t}=0^{+}$, is subject to a time-dependent rotational shear stress. The solutions that have been obtained are presented under series form in terms of Bessel functions, satisfy all imposed initial and boundary conditions. Moreover, these solutions satisfy both the governing differential equations and all imposed initial and boundary conditions. The corresponding solutions for Maxwell, second grade and Newtonian fluids are obtained as limiting case of general solutions. Finally, the influence of the pertinent parameters on the velocity and shear stress of the fluid is analyzed by graphical illustrations.
\end{abstract}

Keywords: Taylor-Couette Flow, Oldroyd-B Fluid, Velocity Field, Shear Stress

\section{Introduction}

Due to the several technological applications the flow analysis of non-Newtonian fluids is very important in the fields of fluid mechanics. Many investigators have not studied the flow behavior of non-Newtonian fluids in various flow fields due to the complex stress - strain relationship [1]. The study of non-Newtonian fluids has got much attention because of their practical applications. Non-Newtonian characteristics are displayed by a number of industrially important fluids including polymers, molten plastic, pulps, microfluids and food stuff display. Exact analytic solutions for the flows of non-Newtonian fluids are important provided they correspond to physically realistic problems and they can be used as checks against complicated numerical codes that have been developed for much more complex flows. Many non-Newtonian models such as differential type, rate type and integral type fluids have been proposed in recent years. Among them, the rate type fluid models have received special attention. The differential type fluids do not predict stress relaxation and they are not successful for describing the flows of some polymers.

Here, we shall consider a model due to Oldroyd [2], which contains as special cases Maxwell, second grade and Newtonian Models. For Newtonian fluid, the velocity field for a fluid contained in an annular region between two co-axial circular cylinders, is given in [3]. The first exact solutions for motions of Oldroyd-B fluids in cylindrical domain seem to be those of Waters and King [4]. In the meantime many papers regarding such motions have been published but we mention here only a few of those regarding Oldroyd-B or more general fluid [5-16].

To the best of our knowledge, the first exact solutions for motions of non-Newtonian fluids, due to a constant shear stress on the boundary, are those of Waters and King [17] over an infinite plate, and Bandelli and Rajagopal [18] between two co-axial circular cylinders. Similar solutions for the flow due to an infinite plate that applies a constant/time-dependent shear to a non-Newtonian fluid, have been also obtained by Erdogan [19], Fetecau and Kannana [20], Awan et al. [21] and Kamran et al. [22]. The computer techniques make the complete integration of the momentum equation feasible, the accuracy of the numerical results can be established by 
comparison with an exact solution. Consequently, as in the case of the motion problems in which the velocity is given on the boundary, it is necessary to develop a large class of exact and approximate solutions for problems in which the boundary (or a part of the boundary) applies a shear stress to the fluid.

The purpose of this paper is to establish exact solutions corresponding to the motion of an Oldroyd-B fluid between two co-axial circular cylinders, in which outer cylinder being fixed and the inner cylinder applying a time-dependent rotational shear stress to the fluid. The Oldroyd-B fluids store energy as linearized elastic solids and their dissipation is due to two dissipative mechanisms which arise from a mixture of two viscous fluids. They have been extensively used in many applications although an Oldroyd-B fluid cannot describe either shear thinning or shear thickening. However, they can describe stress-relaxation, creep and the normal stress differences that develop during simple shear flows. This model is viewed as one of the most successful models for describing the response of a subclass of polymeric liquids. It is worthy to point out that the solutions that have been obtained satisfy both the governing differential equations as well as all imposed initial and boundary conditions. The solutions corresponding to Maxwell, second grade and Newtonian fluids, performing the same motion, are also obtained as limiting cases of general solutions. Finally, the influence of the pertinent parameters on the fluid motion, as well as a comparison between the four models, is shown by graphical illustrations.

\section{Constitutive and Governing Equations}

The Cauchy stres $\mathrm{T}$ for an incompressible Oldroyd-B fluid is related to the fluid motion by the following constitutive equations

$$
\begin{aligned}
& \mathrm{T}=-p \mathrm{I}+\mathrm{S}, \\
& \mathrm{S}+\lambda\left(\mathrm{S}-\mathrm{LS}-\mathrm{SL}^{\tau}\right)=\mu\left[\mathrm{A}+\lambda\left(\mathrm{A}-\mathrm{LA}-\mathrm{AL}^{\tau}\right)\right],
\end{aligned}
$$

where $-p$ I denotes the indeterminate spherical stress due to the constraint of incompressibility, $\mathrm{S}$ is the extra-stress tensor, $\mathrm{L}$ is the velocity gradient $\mathrm{A}=\mathrm{L}+\mathrm{L}^{T}$ is the first Rivlin-Ericksen tensor, $\mu$ is the dynamic viscosity of the fluid, $\lambda$ and $\lambda_{r}(<\lambda)$ are relaxation and retardation times, the superposed dot indicates the material time derivative and the superscript $T$ denotes the transpose operation. The model characterized by Eqs. (1) contains as special cases the upper convected Maxwell model for $\lambda_{r}=0$ and the Newtonian fluid model for $\lambda=\lambda_{r}=0$. In some special flows, like those to be considered here, the governing equations corresponding to the Oldroyd-B fluids resemble those of second grade fluids. Consequently, it is to be expected that the general solutions for Oldroyd-B fluids contain as special cases both the solutions corresponding to Maxwell and Newtonian fluids and those for second grade fluids.

For the problem under consideration we assume a velocity field $\mathrm{V}$ and an extra-stress tensor $\mathrm{s}$ of the form as studied by
Bandeli and Rajagopal [18].

$$
\mathrm{V}=\mathrm{V}(r, t)=w(r, t) \mathrm{e}_{\theta}, \quad \mathrm{S}=\mathrm{S}(r, t),
$$

where $\mathrm{e}_{\theta}$ is the unit vector in the $\theta$-direction of the cylindrical coordinates system $r, \theta$ and $z$. For such flows the constraint of incompressibility is automatically satisfied. If the fluid is at rest up to the moment $t=0$, then

$$
v(r, 0)=0, \mathrm{~S}(r, 0)=0,
$$

and Eqs. (1) $)_{2}$ and (2) imply $S_{r r}=S_{r z}=S_{z z}=S_{z \theta}=0$ and we obtain the meaningful equation $[5,6,13]$

$$
\left(1+\lambda \frac{\partial}{\partial t}\right) \tau(r, t)=\mu\left(1+\lambda_{r} \frac{\partial}{\partial t}\right)\left(\frac{\partial}{\partial r}-\frac{1}{r}\right) w(r, t),
$$

where $\tau=S_{r \theta}$ is the non zero shear stress.

Neglecting body forces and in the absence of a pressure gradient in the axial direction, the balance of the linear momentum leads to the relevant equation [5]

$$
\rho \frac{\partial w(r, t)}{\partial t}=\left(\frac{\partial}{\partial r}+\frac{2}{r}\right) \tau(r, t)
$$

where $\rho$ is the constant density of the fluid. Eliminating $\tau(r, t)$ between Eqs. (4) and (5), the governing equation for velocity is

$$
\left(1+\lambda \frac{\partial}{\partial t}\right) \frac{\partial w(r, t)}{\partial t}=\left(v+\alpha \frac{\partial}{\partial t}\right) \times\left(\frac{\partial^{2}}{\partial r^{2}}+\frac{1}{r} \frac{\partial}{\partial r}-\frac{1}{r^{2}}\right) w(r, t)(6)
$$

where $v=\mu / \rho$ is the kinematic viscosity of the fluid and $\alpha=v \lambda_{r}$.

The partial differential equations (4) and (6), with suitable initial and boundary conditions, can be solved in principle by several methods, their efficiency depending on the domain definition. The integral transforms technique represents a systematic, efficient and powerful tool. We will use Hankel transform to eliminate the spatial variable. In order to avoid the lengthy and burdensome calculations of residues and contour integrals, we shall use the finite Hankel transform.

\section{Rotational Flow Through an Annulus}

Consider an incompressible Oldroyd-B fluid at rest in an annular region between two infinitely long co-axial circular cylinders. After time $t=0$ let the inner cylinder of radius $R_{1}$ be set in rotation about its axis by a time-dependent torque per unit length $2 \pi R_{1} \tau\left(R_{1}, t\right)$, where

$$
\tau\left(R_{1}, t\right)=f\left[t-\lambda\left(1-e^{-t / \lambda}\right)\right] ; f=\text { constant }
$$

and let the outer cylinder of radius $R_{2}\left(>R_{1}\right)$ be held fixed. Owing to the shear the fluid between cylinders is gradually moved. Its velocity is of the form (2) , the governing equations are given by Eqs. (4) and (6) while the appropriate 
initial and boundary conditions are

$$
\begin{aligned}
& w(r, 0)=\frac{\partial w(r, 0)}{\partial t}=0, \tau(r, 0)=0 ; r \in\left(R_{1}, R_{2}\right], \\
&\left.\left(1+\lambda \frac{\partial}{\partial t}\right) \tau(r, t)\right|_{r=R_{1}}=\mu\left(1+\lambda_{r} \frac{\partial}{\partial t}\right) \\
& \quad \times\left.\left(\frac{\partial}{\partial r}-\frac{1}{r}\right) w(r, t)\right|_{r=R_{1}}=f t ; \quad w\left(R_{2}, t\right)=0 ; t>0 .
\end{aligned}
$$

The $\tau\left(R_{1}, t\right)$ given by Eq. (7) is just the solution of the differential equation $(9)_{1}$.

\subsection{Calculation of the Velocity Field}

We shall denote by $[23,24]$

$$
w_{n H}\left(r_{n}, t\right)=\int_{R_{1}}^{R_{2}} r w(r, t) B\left(r, r_{n}\right) d r,
$$

the finite Hankel transform of $w(r, t)$, where

$$
B\left(r, r_{n}\right)=J_{1}\left(r r_{n}\right) Y_{2}\left(R_{1} r_{n}\right)-J_{2}\left(R_{1} r_{n}\right) Y_{1}\left(r r_{n}\right),
$$

where $J_{p}(\cdot)$ and $Y_{p}(\cdot)$ are the Bessel functions of the first and second kind of order $p$ and $r_{n}$ are the positive roots of the transcendental equation $B\left(R_{2}, r\right)=0$.

The inverse Hankel transform of $w_{n H}\left(r_{n}, t\right)$ is given by $[23,24]$

$$
w(r, t)=\frac{\pi^{2}}{2} \sum_{n=1}^{\infty} \frac{r_{n}^{2} J_{1}^{2}\left(R_{2} r_{n}\right) B\left(r, r_{n}\right)}{J_{2}^{2}\left(R_{1} r_{n}\right)-J_{1}^{2}\left(R_{2} r_{n}\right)} w_{n H}\left(r_{n}, t\right) .
$$

Now Multiplying Eq. (6) by $r B\left(r, r_{n}\right)$, then integrating it with respect to $\mathrm{r}$ from $R_{1}$ to $R_{2}$, and using the identity

$$
w(r, t)=\frac{f t}{2 \mu}\left(\frac{R_{1}}{R_{2}}\right)^{2}\left(r-\frac{R_{2}^{2}}{r}\right)-\frac{\pi f}{\mu r_{n}} \sum_{n=1}^{\infty} \frac{J_{1}^{2}\left(R_{2} r_{n}\right) B\left(r, r_{n}\right)}{\left[J_{2}^{2}\left(R_{1} r_{n}\right)-J_{1}^{2}\left(R_{2} r_{n}\right)\right]}\left[\frac{e^{p_{2 n} t}-e^{p_{1 n} t}}{p_{2 n}-p_{1 n}}+\frac{1+\alpha r_{n}^{2}}{v r_{n}^{2}}\left(1-\frac{p_{2 n} e^{p_{1 n} t}-p_{1 n} e^{p_{2 n} t}}{p_{2 n}-p_{1 n}}\right)\right]
$$

it can be written in simpler form as

$$
\begin{aligned}
& w(r, t)=\frac{f}{2 \mu}\left(\frac{R_{1}}{R_{2}}\right)^{2}\left(r-\frac{R_{2}^{2}}{r}\right)\left(t-\lambda_{r}\right)-\frac{\pi f}{\mu \nu} \sum_{n=1}^{\infty} \frac{J_{1}^{2}\left(R_{2} r_{n}\right) B\left(r, r_{n}\right)}{r_{n}^{3}\left[J_{2}^{2}\left(R_{1} r_{n}\right)-J_{1}^{2}\left(R_{2} r_{n}\right)\right]} \\
& -\sum_{n=1}^{\infty} \frac{J_{1}^{2}\left(R_{2} r_{n}\right) B\left(r, r_{n}\right)}{r_{n}^{3}\left[J_{2}^{2}\left(R_{1} r_{n}\right)-J_{1}^{2}\left(R_{2} r_{n}\right)\right]}\left[\frac{e^{p_{2 n} t}-e^{p_{1 n} t}}{p_{2 n}-p_{1 n}}-\frac{1+\alpha r_{n}^{2}}{v r_{n}^{2}}\left(\frac{p_{2 n} e^{p_{1 n} t}-p_{1 n} e^{p_{2 n} t}}{p_{2 n}-p_{1 n}}\right)\right],
\end{aligned}
$$

or equivalently

$$
w(r, t)=\frac{f}{2 \mu}\left(\frac{R_{1}}{R_{2}}\right)^{2}\left(r-\frac{R_{2}^{2}}{r}\right)\left(t-\lambda_{r}\right)-\frac{\pi f}{\mu \nu} \sum_{n=1}^{\infty} \frac{J_{1}^{2}\left(R_{2} r_{n}\right) B\left(r, r_{n}\right)}{r_{n}^{3}\left[J_{2}^{2}\left(R_{1} r_{n}\right)-J_{1}^{2}\left(R_{2} r_{n}\right)\right]} \times\left(1-\lambda \frac{p_{1 n}^{2} e^{p_{2 n} t}-p_{2 n}^{2} e^{p_{1 n} t}}{p_{2 n}-p_{1 n}}\right) .
$$

\subsection{Calculation of the Shear Stress}

Solving partial differential equation (4) with respect to $\tau(r, t)$ and using the initial condition $(8)_{3}$, we find that 


$$
\tau(r, t)=\frac{\mu}{\lambda} e^{-\frac{t}{\lambda}} \int_{0}^{t} e^{\frac{s}{\lambda}}\left(1+\lambda_{r} \frac{\partial}{\partial s}\right)\left(\frac{\partial}{\partial r}-\frac{1}{r}\right) w(r, s) d s
$$

Substituting $w(r, t)$ from Eq. (20) into Eq. (21) we get after lengthy but straightforward computations the simple form of the shear stress

$$
\tau(r, t)=f\left[t-\lambda\left(1-e^{-t / \lambda}\right)\right]\left(\frac{R_{1}}{r}\right)^{2}+\frac{\pi f}{v} \sum_{n=1}^{\infty} \frac{J_{1}^{2}\left(R_{2} r_{n}\right) B^{*}\left(r, r_{n}\right)}{r_{n}^{2}\left[J_{2}^{2}\left(R_{1} r_{n}\right)-J_{1}^{2}\left(R_{2} r_{n}\right)\right]} \times\left[1-\frac{p_{2 n} e^{p_{1 n} t}-p_{1 n} e^{p_{2 n} t}}{p_{2 n}-p_{1 n}}\right],
$$

where $B^{*}\left(r, r_{n}\right)=J_{2}\left(r r_{n}\right) Y_{2}\left(R_{1} r_{n}\right)-J_{2}\left(R_{1} r_{n}\right) Y_{2}\left(r r_{n}\right)$.

\section{Limiting Cases}

\subsection{Maxwell Fluid}

Taking the limit as $\lambda_{r} \rightarrow 0$ in Eqs. (20) and (22), we recover the solutions

$$
\begin{gathered}
w_{M}(r, t)=\frac{f t}{2 \mu}\left(\frac{R_{1}}{R_{2}}\right)^{2}\left(r-\frac{R_{2}^{2}}{r}\right)-\frac{\pi f}{\mu v} \sum_{n=1}^{\infty} \frac{J_{1}^{2}\left(R_{2} r_{n}\right) B\left(r, r_{n}\right)}{r_{n}^{3}\left[J_{2}^{2}\left(R_{1} r_{n}\right)-J_{1}^{2}\left(R_{2} r_{n}\right)\right]} \times\left(1-\lambda \frac{p_{3 n}^{2} e^{p_{4 n} t}-p_{4 n}^{2} e^{p_{3 n} t}}{p_{4 n}-p_{3 n}}\right), \\
\tau_{M}(r, t)=f\left[t-\lambda\left(1-e^{-t / \lambda}\right)\right]\left(\frac{R_{1}}{r}\right)^{2}+\frac{\pi f}{v} \sum_{n=1}^{\infty} \frac{J_{1}^{2}\left(R_{2} r_{n}\right) B^{*}\left(r, r_{n}\right)}{r_{n}^{2}\left[J_{2}^{2}\left(R_{1} r_{n}\right)-J_{1}^{2}\left(R_{2} r_{n}\right)\right]} \times\left[1-\frac{p_{4 n} e^{p_{3 n} t}-p_{3 n} e^{p_{4 n} t}}{p_{4 n}-p_{3 n}}\right],
\end{gathered}
$$

corresponding to a Maxwell fluid performing the same motion obtained by Jamil and Fetecau [25] by using different technique. In the above relations

$$
p_{3 n}, p_{4 n}=\frac{-1 \pm \sqrt{1-4 v \lambda r_{n}^{2}}}{2 \lambda}
$$

\subsection{Second Grade Fluid}

When $\lambda \rightarrow 0$ in Eqs. (20) and (22), we obtain the solutions

$$
\begin{gathered}
w_{S G}(r, t)=\frac{1}{2 \mu}\left(\frac{R_{1}}{R_{2}}\right)^{2}\left(r-\frac{R_{2}^{2}}{r}\right) f\left(t-\lambda_{r}\right)-\frac{\pi f}{\mu \nu} \sum_{n=1}^{\infty} \frac{J_{1}^{2}\left(R_{2} r_{n}\right) B\left(r, r_{n}\right)}{r_{n}^{3}\left[J_{2}^{2}\left(R_{1} r_{n}\right)-J_{1}^{2}\left(R_{2} r_{n}\right)\right]} \times\left\{1-\left(1+\alpha r_{n}^{2}\right) \exp \left(-\frac{v r_{n}^{2} t}{1+\alpha r_{n}^{2}}\right)\right\}, \\
\tau_{S G}(r, t)=\left(\frac{R_{1}}{r}\right)^{2} f t+\frac{\pi f}{v} \times \sum_{n=1}^{\infty} \frac{J_{1}^{2}\left(R_{2} r_{n}\right) B^{*}\left(r, r_{n}\right)}{r_{n}^{2}\left[J_{2}^{2}\left(R_{1} r_{n}\right)-J_{1}^{2}\left(R_{2} r_{n}\right)\right]}\left(1-\exp \left(\frac{-v r_{n}^{2} t}{1+\alpha r_{n}^{2}}\right)\right),
\end{gathered}
$$

corresponding to a second grade fluid. Eq. (25) can also be written in the following form

$$
w_{S G}(r, t)=\frac{1}{2 \mu}\left(\frac{R_{1}}{R_{2}}\right)^{2}\left(r-\frac{R_{2}^{2}}{r}\right) f t-\frac{\pi f}{\mu v} \sum_{n=1}^{\infty} \frac{J_{1}^{2}\left(R_{2} r_{n}\right) B\left(r, r_{n}\right)}{r_{n}^{3}\left[J_{2}^{2}\left(R_{1} r_{n}\right)-J_{1}^{2}\left(R_{2} r_{n}\right)\right]} \times\left(1+\alpha r_{n}^{2}\right)\left(1-\exp \left(-\frac{v r_{n}^{2} t}{1+\alpha r_{n}^{2}}\right)\right) .
$$

\subsection{Newtonian Fluid}

Finally when $\lambda \rightarrow 0$ in Eqs. (23) and (24) or $\lambda_{r} \rightarrow 0$ and then $\alpha \rightarrow 0$ into Eqs. (25) and (26), the solutions

$$
\begin{gathered}
w_{N}(r, t)=\frac{1}{2 \mu}\left(\frac{R_{1}}{R_{2}}\right)^{2}\left(r-\frac{R_{2}^{2}}{r}\right) f t-\frac{\pi f}{\mu \nu} \times \sum_{n=1}^{\infty} \frac{J_{1}^{2}\left(R_{2} r_{n}\right) B\left(r, r_{n}\right)}{r_{n}\left[J_{2}^{2}\left(R_{1} r_{n}\right)-J_{1}^{2}\left(R_{2} r_{n}\right)\right]}\left(1-\exp \left(-v r_{n}^{2} t\right)\right), \\
\tau_{N}(r, t)=\left(\frac{R_{1}}{r}\right)^{2} f t+\frac{\pi f}{v} \times \sum_{n=1}^{\infty} \frac{J_{1}^{2}\left(R_{2} r_{n}\right) B^{*}\left(r, r_{n}\right)}{J_{2}^{2}\left(R_{1} r_{n}\right)-J_{1}^{2}\left(R_{2} r_{n}\right)}\left(1-\exp \left(-v r_{n}^{2} t\right)\right) .
\end{gathered}
$$


for a Newtonian fluid are recovered.

Of course when $\lambda \rightarrow 0$ in Eq. (7), we find that

$$
\tau\left(R_{1}, t\right)=f t .
$$

Consequently, the solutions (25) and (26), as well as (28) and (29), correspond to a couple $f t$ on the boundary.

\section{Numerical Results and Conclusions}

The purpose of this paper was to provide exact solutions for the velocity field $w(r, t)$ and the shear stress $\tau(r, t)$ corresponding to the flow of an Oldroyd-B fluid between two infinite co-axial cylinders, the inner cylinder being subject to a time-dependent torque. The solutions that have been obtained, presented under series form in terms of Bessel functions $J_{1}(\cdot)$, $J_{2}(\cdot), Y_{1}(\cdot)$ and $Y_{2}(\cdot)$, satisfy both the governing equations and all imposed initial and boundary conditions. They can easily be simplified to give similar solutions for Maxwell, second grade and Newtonian fluids. The solutions for second grade and Newtonian fluids correspond to a time dependent torque $f t$ on the boundary.

In order to exhibit some relevant physical aspects of the obtained results, the diagrams of the velocity $w(r, t)$ and of the shear stress $\tau(r, t)$ are plotted against $r$ for different values of $t$ and of the pertinent parameters. From Figs. $1 a$ and $1 b$ the influence of the rigid boundary on the fluid motion is clearly evident. The velocity of the fluid, as well as the shear stress in absolute value, is an increasing function with respect to $t$ and a decreasing one with respect to $r$. The veolcity is

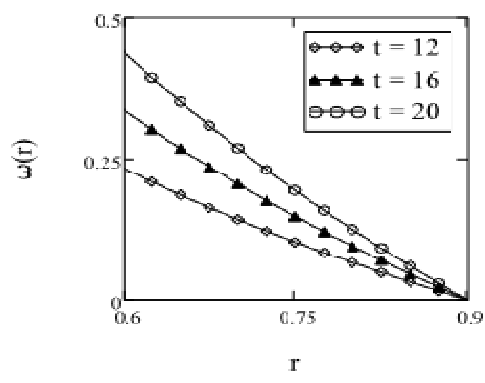

(a) decreasin with respect $r$ due to the fact that we are applying time depemdent stress on the inner cylinder. This shows that our solution satisfy the boundary coditions as the velocity on the outer cylinder is zero. In Figs. 2, it is shown the influence of the kinematic viscosity $v$ on the fluid motion. It is clearly seen that the velocity as well as the shear stress in absolute value, is a decreasing function with respect to $v$. It is due the fact that velocity of fluid decreases as the fluid become ore thick, as the incrasing value of viscosity give more resistance to fluid to flow. Figs. 3 and 4 show the influence of the relaxation and retardation times $\lambda$ and $\lambda_{r}$ on the fluid motion. The two parameters, as it was to be expected, have opposite effects on the fluid motion. Both the velocity and the shear stress (in absolute value) are decreasing functions with respect to $\lambda$ and increasing ones with regard to $\lambda_{r}$.

Finally, for comparison, the diagrams of $w(r, t)$ corresponding to the four models (Oldroyd-B, Maxwell, second grade and Newtonian) are presented in Figs. 5 for the same values of the common pertinent parameters and the time $t$. In all cases the velocity of the fluid is a decreasing function with respect to $r$. The Newtonian fluid as it results from Fig. $5(\mathrm{a})$, is the swiftest on the whole flow domain while the Oldroyd-B fluid is the slowest excepting a small region near the outer cylinder. The comparison of Figs. 5 (a) and (b), clearly shows that for large values of time, Oldroyd-B, Maxwell and second grade fluid tends to Newtonian fluid. Consequently the non-Newtonian effects disappear for $t \rightarrow \infty$. The units of the parameters into Figs. 1-5, are SI units and the roots $r_{n}$ have been approximated by $(2 n-1) \pi /\left[2\left(R_{2}-R_{1}\right)\right]$.

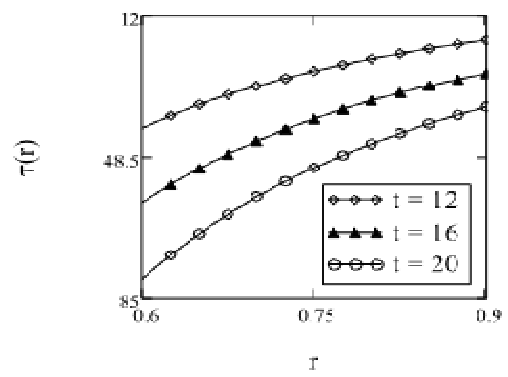

(b)

Figure 1. Profiles of the velocity ${ }^{w}(r, t)$ and shear stress $\tau(r, t)$ given by Eqs. (20) and (22) for $R_{1}=0.6, R_{2}=0.9, f=-5, \quad v=0.57, \mu=32, \lambda=4, \lambda=3$ and different values of $t$.

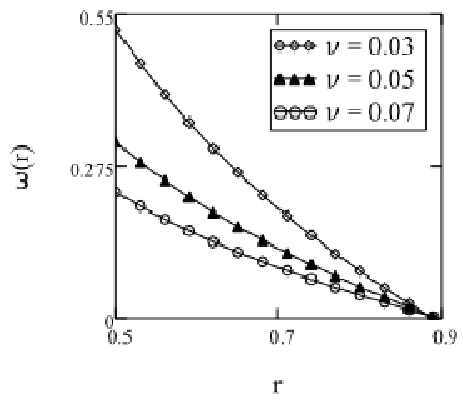

(a)

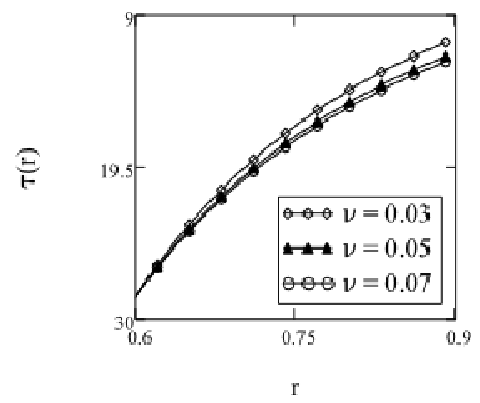

(b)

Figure 2. Profiles of the velocity $w(r, t)$ and shear stress $\tau(r, t)$ given by Eqs. (20) and (22) for $R_{1}=0.6, R_{2}=0.9, f=-5, \quad t=10, \lambda=5, \lambda_{r}=1$ and different values of $v$. 


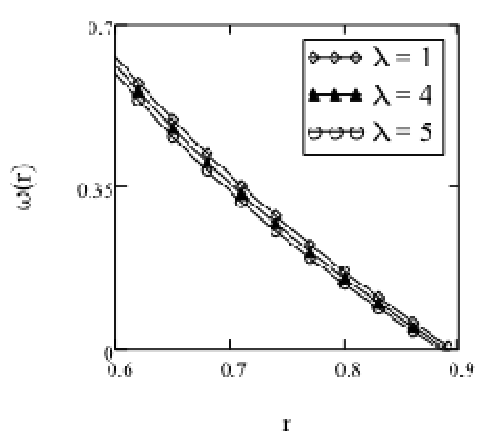

(a)

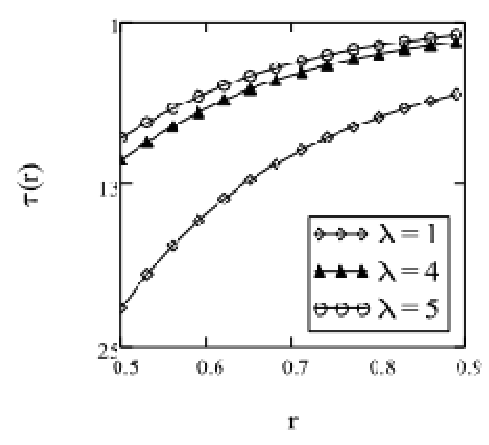

(b)

Figure 3. Profiles of the velocity $w(r, t)$ and shear stress $\tau(r, t)$ given by Eqs. (20) and (22) for $R_{1}=0.6, R_{2}=0.9, f=-5, \quad t=4, v=0.03575, \mu=5, \lambda_{r}=0.1$ and different values of $\lambda$.

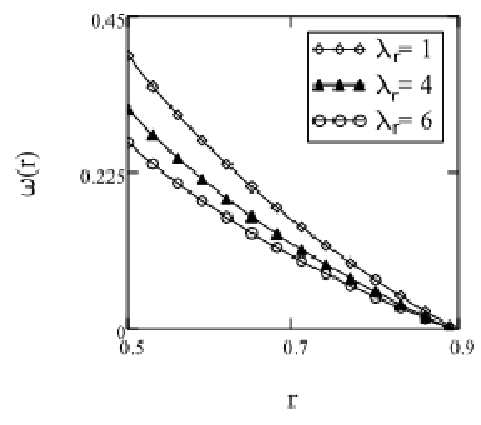

(a)

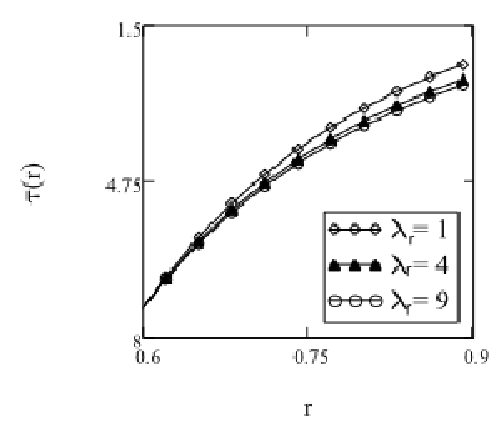

(b)

Figure 4. Profiles of the velocity $w(r, t)$ and shear stress $\tau(r, t)$ given by Eqs. (20) and (22) for $R_{1}=0.6, R_{2}=0.9, t=17, \quad f=-2, \lambda=10, v=0.025, \mu=32 \quad$ and different values of $\lambda$.

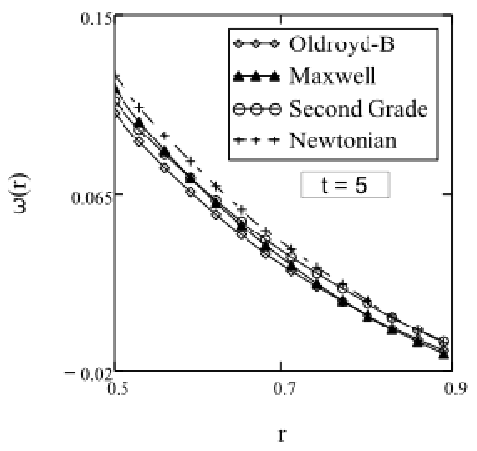

(a)

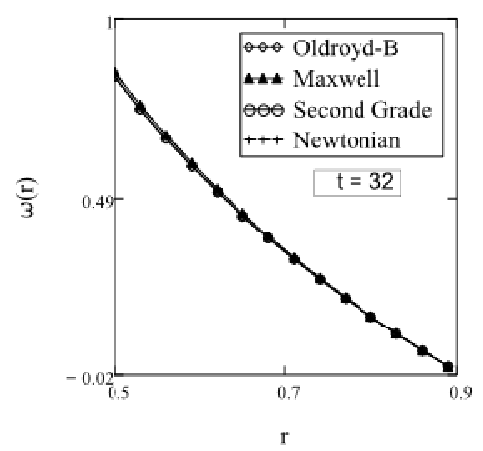

(b)

Figure 5. Profiles of the velocity $w(r, t)$ corresponding to the Newtonian, second grade, Maxwell and Oldroyd-B fluids for $R_{1}=0.5, R_{2}=0.9, f=-5, \lambda=3, \lambda_{r}=0.5, \mu=32$ and $v=0.025$.

\section{Acknowledgement}

The authors are extremely grateful and thankful to the Department of Mathematics, GC University, Faisalabad. Pakistan and Higher Education Commission of Pakistan for supporting and facilitating this research work.

\section{References}

[1] S. F. Han, Constitutive equation and computational analytical theory Of non-Newtonian fluids, Science Press, Beijing (2000).
[2] J. G. Oldroyd, On the formulation of rheological equations of state, Proc. Roy. Soc., London Ser. A 38, pp.. 523-541, 1950.

[3] C. S. Yih, Fluid Mechanics, McGraw Hill, New York 1969.

[4] N. D. Waters and M. J. King, The unsteady flow of an elasto-viscous liquid in a straight pipe of circular cross section, J. Phys. D: App.1. Phys. 4, pp. 204-211, 1971.

[5] K. R. Rajagopal, R. K. Bhatnagar, Exact solutions for some simple flows of Oldroyd-B fluid, Acta Mech. 113, pp. 233-239, 1995.

[6] W. P. Wood, Transient viscoelastic helical flows in pipes of circular and annular cross-section, J. Non-Newtonian Fluid 
Mech. 100, pp. 115-126, 2001.

[7] M. H. Haroun, On non-linear magnetohydrodynamics flow due to peristaltic transport of an Oldroyd 3-constant fluid, $\mathrm{Z}$. Naturforsch. 61a No. 5, pp. 263-274, 2006.

[8] T. Hayat, M. Khan, and T. Wang, Non-Newtonian flow between concentric cylinders, Commun. Nonlinear Sci. Numer. Simul. 11, pp. 297-305, 2006.

[9] C. Fetecau, Corina Fetecau, D. Vieru, On some helical flows of Oldroyd-B fluids, Acta Mech. 189, pp. 53-63, 2007.

[10] Corina Fetecau, T. Hayat, and C. Fetecau, Starting solutions for oscillating motions of Oldroyd-B fluids in cylindrical domains, J. Non-Newtonian Fluid Mech. 153, pp. 191-201 , 2008.

[11] R. Ellahi, T. Hayat, T. Javed, S. Asghar, On the analytic solution of nonlinear flow problem involving Oldroyd 8-constant fluid, Math. Comput. Model. 48, pp. 1191-1200, 2008.

[12] Corina Fetecau, C. Fetecau, M. Imran, Axial Couette flow of an Oldroud-B fluid due to a time-dependent shear stress, Math. Reports 11 (61), No. 2, pp. (2009) 145-154.

[13] Corina Fetecau, M. Imran, C. Fetecau, I. Burdujan, Helical flow of an Oldroyd-B fluid due to a circular cylinder subject to time dependent shear stresses, Z. Angew. Math. Phys., 61, pp. 959-969, 2010.

[14] R. Ellahi, T. Hayat, F. M. Mahomed, and A. Zeeshan, Exact solutions for flows of an Oldroyd 8-constant fluid with nonlinear slip conditions, Commun. Nonlinear Sci. Numer. Simul. 15 , pp. 322-330, 2010.

[15] T. Hayat, S. Najam, M. Sajid, and M. Ayub, On exact solutions of oscillatory flows in a generalized Burger's fluid with slip condition, Z. Naturforsch. 65a No. 4, pp. 381-390, 2010.

[16] Corina Fetecau, M. Imran And C. Fetecau, Taylor-Couette flow of an Oldroyd-B fluid in an annulus due to a time-dependent couple, Zeit. Nat. A 66a, pp. 40-46, 2011.

[17] N. D. Waters and M. J. King, Unsteady flow of an elastico-viscous liquid, Rheol. Acta 9, pp. 345--355, 1970.

[18] R. Bandelli, K. R. Rajagopal, Start-up flows of second grade fluids in domains with one finite dimension, Int. J. Non-Linear Mech. 30, pp. 817-839, 1995.

[19] M. E. Erdogan, On unsteady motions of fluids of a second-order fluid, Int. J. Non-Linear Mech. 38, pp. 1045-1051, 2003.

[20] C. Fetecau and K. Kannan, A note on an unsteady flow of an Oldroyd-B fluid, Int. J. Math. Math. Sci. 19, pp. 3185-3194, 2005.

[21] A. U. Awan, Corina Fetecau, Qammar Rubbab, Axial Couette flow of a generalized Oldroyd-B fluid due to a longitudinal time-dependent shear stress, Quaestiones Mathematicae, 33, pp. 429-441, 2010.

[22] M. Kamran , M. Imran, M. Athar , M. A. Imran, On the unsteady rotational flow of fractional Oldroyd-B fluid in cylindrical domains, Meccanica, DOI 10.1007/s11012-011-9467-4.

[23] Tong, D., Liu, Y., Exact solutions for the unsteady rotational flow of non-Newtonian fluid in an annular pipe, Int. J. Eng. Sci. 43, pp. 281-289, 2005.

[24] L. Debnath and D. Bhatta, Integral Transforms and their Applications (second ed.), Chapman and Hall/CRC Press, Boca-Raton-London-New York 2007.

[25] M. Jamil, C. Fetecau, Helical flows of Maxwell fluid between two cylinders with given shear stresses on the boundary, Nonlinear Analysis: Real World Applications 11, pp. 4302-4311, 2010. 